\title{
THE ABSORPTION, EXCRETION, AND DISTRIBUTION OF PENICILLIN ${ }^{1}$
}

\author{
By CHARLES H. RAMMELKAMP AND CHESTER S. KEEFER \\ (From the Evans Memorial, Massachusetts Memorial Hospitals, and the Department of \\ Medicine, Boston University School of Medicine, Boston)
}

(Received for publication December 7, 1942)

In 1929, Fleming (1) described an active antibacterial substance, penicillin, obtained from the mold, Penicillium notatum. This substance has been shown to exhibit a marked antibacterial effect both in vitro $(1,2)$ and in the experimental animal (2). In man, the observations on the efficacy of penicillin as a therapeutic agent have been limited to those of Florey and his co-workers (3).

As a part of our investigation of the therapeutic effectiveness of penicillin in various infections, this study was undertaken to determine its absorption, excretion, and distribution when administered by various routes.

\section{MATERIALS AND METHODS}

The subjects included normal volunteers and ward patients. The latter group were for the most part suffering from localized infections. Unless otherwise indicated, the urine and the blood non-protein nitrogen were normal in each subject. The majority of the studies were made in the fasting state; however, after the first 4 hours of observation fluids were not limited.

Penicillin 2 in the form of the sodium salt was dissolved in either distilled water or 0.85 per cent sodium chloride solution and passed through a Seitz filter to effect sterilization. The final concentration of all solutions of penicillin was 1,000 Florey units per cubic centimeter except that administered subcutaneously, which contained 200 Florey units per cubic centimeter of 0.85 per cent sodium chloride. The various solutions of penicillin were stored at $5^{\circ} \mathrm{C}$. until time of use.

Penicillin was administered by the oral, intraduodenal, rectal, intravenous, subcutaneous, intramuscular, intrapleural, intra-articular, and intrabursal routes. The oral and rectal doses were administered in $200 \mathrm{cc}$. of tap water. Prior to rectal administration the subject was given a soap-and-water enema. Intraduodenal administration was effected through a Miller-Abbott tube, the position being checked by fluoroscopic examination. Intraarticular and intrabursal injections were made after aspi-

\footnotetext{
1 Supported by a grant from the Johnson Research Foundation, New Brunswick, New Jersey.

2 The penicillin used in this study was supplied through the courtesy of Dr. George A. Harrop, Squibb Institute for Medical Research, New Brunswick, New Jersey.
}

ration of a transudate. The gluteal muscles were used for intramuscular injections, and the medial aspect of the thigh for subcutaneous injections. Intrapleural injections were made in subjects with empyema, the exudate having been aspirated just prior to the administration of penicillin.

No serious toxic manifestations were caused by the administration of penicillin during the course of this study. The subjects complained of no untoward symptoms after intravenous injections of 5,000 to 40,000 Florey units. In one subject who received a constant intravenous drip of 102,500 Florey units in a period of 38 hours, no toxic reaction occurred. The intramuscular injection of 10,000 Florey units of penicillin in distilled water was attended by definite residual soreness, but such reactions were not encountered when the penicillin was dissolved in 0.85 per cent sodium chloride. The subcutaneous injection of 10,000 Florey units in $50 \mathrm{cc}$. of 0.85 per cent sodium chloride resulted in soreness and erythema, and the latter did not disappear for 12 to 24 hours. When a somewhat more dilute solution of penicillin was used, no erythema was noted. Penicillin has a very bitter taste, so that oral administration was somewhat unpleasant.

Samples of blood were withdrawn from each subject before and at frequent intervals after the dose of penicillin had been administered. The blood was then either defibrinated ${ }^{3}$ or allowed to clot in sterile tubes. In both instances, the serum was separated by centrifugalization.

Urine was collected in a sterile container from all male subjects. In females, it was usually voided without sterile precautions except in a few subjects from whom the urine was obtained every 15 minutes by means of an inlying catheter. In general, for a period of 24 hours after the penicillin had been given, all urine was collected as individual specimens.

Spinal fluid was obtained at varying intervals up to 195 minutes after the injection of penicillin. Joint fluid and exudate from the pleural cavity were obtained by aspiration.

All samples of body fluids were stored at $5^{\circ} \mathrm{C}$., without the addition of any preservative, until the time of testing. If the sample was known to be contaminated, it was passed through a Seitz filter. In most cases, the determinations of penicillin were made on the same day the subject received the material. In a few subjects, the sam-

3 The blood was defibrinated in many cases because a simultaneous study was made on the bactericidal power of whole blood following penicillin administration. This study will be reported at a later date. 
ples were tested several days after the specimens were obtained.4

The method used to determine the concentration of penicillin in the samples of the various body fluids has been described previously (4). In brief, serial dilutions of $0.2 \mathrm{cc}$. of the unknown sample were made with $0.2 \mathrm{cc}$. plain broth through a series of 3 to 14 tubes. In addition, $0.5 \mathrm{cc}$. of the unknown sample was added to one tube. Similar dilutions of a known standard of penicillin, containing 20 Florey units per cc., were made. To all the tubes was added, then, $0.5 \mathrm{cc}$. of plain broth containing 1 per cent erythrocytes and from 1,000 to 10,000 hemolytic streptococci of a standard strain. The tubes were placed in an incubator at $37^{\circ} \mathrm{C}$. for 18 to 24 hours and examined for visible growth. In general, those cultures showing no hemolysis were sterile. However, this was checked by streaking several dilutions on either side of the end point on blood-agar plates.

The concentration of penicillin in the unknown sample may then be determined by comparison with the standard control of penicillin. ${ }^{5}$ It has been found that 0.0039 Florey unit is required to sterilize the culture of hemolytic streptococci contained in a total volume of $0.7 \mathrm{cc}$.

\section{EFFECT OF INTRAVENOUS ADMINISTRATION ON THE SERUM CONCENTRATION AND URINARY} EXCRETION

Figure 1 shows the concentrations of penicillin in the serum, and the cumulative excretion in the urine in a subject with an inlying catheter, following the injection of 20,000 Florey units. The

4 If the specimen to be tested is kept at icebox temperatures, there is no appreciable loss of penicillin (4).

5 The figures recorded in this paper on the concentration of penicillin in the various body fluids are subject to the error of dilution methods in general. level in the serum rose rapidly, reaching a maximum immediately after the injection. Following this, there was a very rapid fall and at the end of 140 minutes, no penicillin could be detected. The rapid excretion into the urine is especially well demonstrated here, where 43 per cent of the injected dose was recovered within 1 hour after the injection. The concentration of penicillin was so high during this period that the urine was colored a bright yellow. In a few patients from whom frequent collections of urine were made, it was also noted that the period of greatest excretion of penicillin was accompanied by an increased volume of urine.

In all experiments in normal subjects, from 37 to 99 per cent of the intravenous dose was found in the urine, and the greatest amount was excreted in the first hour.

The effect on the concentration in the serum of varying the size of the dose of penicillin is illustrated in Figure 2. The single doses ranged from 5,000 to 40,000 Florey units. The greatest rise occurred immediately following the largest dose. Traces of penicillin were detected in the serum for only 30 to 40 minutes after 5,000 Florey units had been injected, whereas with amounts as large as 20,000 to 40,000 units, traces were observed for as long as 185 minutes after injection.

It is of some interest to point out that the spinal fluid of 3 patients (Subjects 4, 5, and 8, Table I) contained no penicillin after the injection of from 10,000 to 20,000 Florey units.

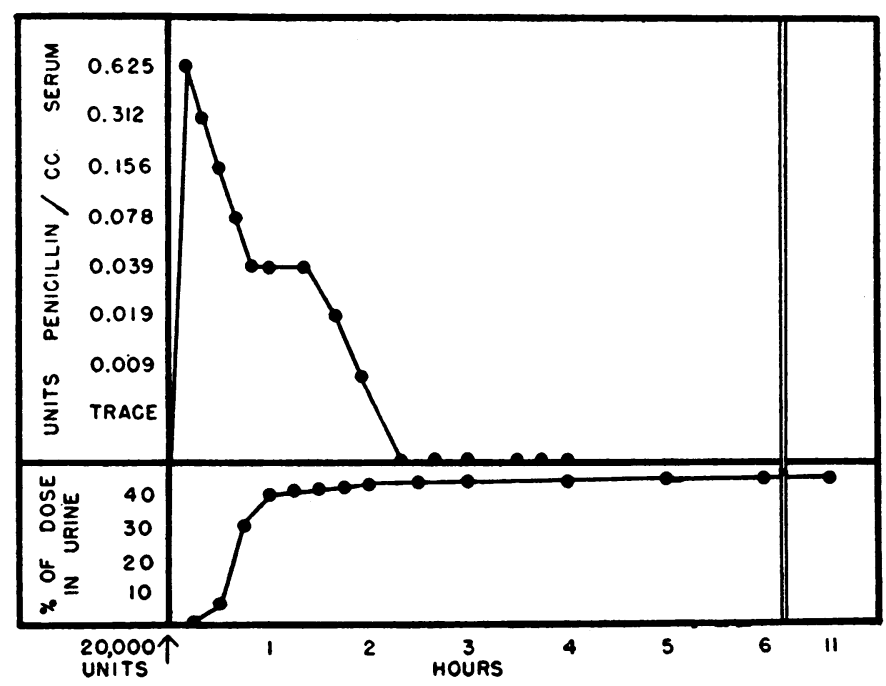

Fig. 1. Rate of Excretion of Penicillin Following Intravenous ADMINISTRATION 
, TABLE I

Serum concentrations and urinary excretion of penicillin after administration by various routes

\begin{tabular}{|c|c|c|c|c|c|c|c|c|c|c|c|}
\hline \multirow[b]{2}{*}{ Route } & \multirow[b]{2}{*}{ Dose } & \multicolumn{3}{|c|}{ Subject } & \multicolumn{2}{|c|}{ Serum } & \multicolumn{4}{|c|}{ Urine } & \multirow[b]{2}{*}{$\begin{array}{l}\text { Diagnosis and } \\
\text { remarks }\end{array}$} \\
\hline & & Number & Age & Weight & Time* & Units & Time & Volume & Units & $\begin{array}{l}\text { Units } \\
\text { ex- } \\
\text { creted } \\
\text { (cumu- } \\
\text { lative) }\end{array}$ & \\
\hline $\begin{array}{l}\text { Intra- } \\
\text { venous }\end{array}$ & 5,000 & 1 & $\begin{array}{l}\text { years } \\
36\end{array}$ & $\begin{array}{l}\text { lbs. } \\
139\end{array}$ & $\begin{array}{c}\text { minutes } \\
5 \\
20 \\
30 \\
40 \\
70\end{array}$ & $\begin{array}{c}\text { per cc. } \\
0.156 \\
0.039 \\
0.019 \\
\text { tr. } \\
0\end{array}$ & minutes & cc. & per cc. & & Chronic osteomyelitis \\
\hline $\begin{array}{l}\text { Intra- } \\
\text { venous }\end{array}$ & 5,000 & 2 & 31 & 192 & $\begin{array}{r}1 \\
3 \\
9 \\
30 \\
40\end{array}$ & $\begin{array}{c}0.625 \\
0.312 \\
0.156 \\
0.078 \\
0\end{array}$ & $\begin{array}{r}60 \\
200 \\
540\end{array}$ & $\begin{array}{l}164 \\
118 \\
440\end{array}$ & $\begin{array}{l}20.0 \\
1.25 \\
0.039\end{array}$ & $\begin{array}{l}3,280 \\
3,427 \\
3,444\end{array}$ & Normal subject \\
\hline $\begin{array}{l}\text { Intra- } \\
\text { venous }\end{array}$ & 5,000 & 11 & 28 & 152 & $\begin{array}{r}1 \\
3 \\
7 \\
12 \\
20 \\
30 \\
40\end{array}$ & $\begin{array}{c}1.25 \\
0.312 \\
0.156 \\
0.156 \\
0.039 \\
\text { tr. } \\
0\end{array}$ & $\begin{array}{r}60 \\
655\end{array}$ & $\begin{array}{r}97 \\
379\end{array}$ & $\begin{array}{l}40.0 \\
0.625\end{array}$ & $\begin{array}{l}3,880 \\
4,117\end{array}$ & Normal subject \\
\hline $\begin{array}{l}\text { Intra- } \\
\text { venous }\end{array}$ & 10,000 & 2 & 31 & 192 & $\begin{array}{r}5 \\
25 \\
50 \\
70 \\
95 \\
110\end{array}$ & $\begin{array}{c}0.625 \\
0.078 \\
0.039 \\
0.019 \\
0.007 \\
0\end{array}$ & $\begin{array}{r}60 \\
120 \\
180 \\
240\end{array}$ & $\begin{array}{r}144 \\
590 \\
59 \\
32\end{array}$ & $\begin{array}{l}40.0 \\
0.625 \\
1.25 \\
1.25\end{array}$ & $\begin{array}{l}5,760 \\
6,127 \\
6,200 \\
6,232\end{array}$ & Normal subject \\
\hline $\begin{array}{l}\text { Intra- } \\
\text { venous }\end{array}$ & 10,000 & 3 & 40 & 118 & $\begin{array}{r}2 \\
10 \\
20 \\
30 \\
37 \\
45 \\
60 \\
75 \\
90 \\
105\end{array}$ & $\begin{array}{c}1.25 \\
0.312 \\
0.156 \\
0.156 \\
0.078 \\
0.039 \\
0.019 \\
0.019 \\
0.007 \\
0\end{array}$ & $\stackrel{5}{240}+$ & $\begin{array}{r}3 \\
245\end{array}$ & $\begin{array}{r}0 \\
16\end{array}$ & $\begin{array}{r}0 \\
3,955\end{array}$ & Normal subject \\
\hline $\begin{array}{l}\text { Intra- } \\
\text { venous }\end{array}$ & 10,000 & 4 & 55 & 111 & $\begin{array}{r}5 \\
30 \\
90 \\
150 \\
210\end{array}$ & $\begin{array}{l}0.625 \\
0.078 \\
0.019 \\
0.019 \\
0.019\end{array}$ & & & & & $\begin{array}{l}\text { Spinal fluid at } 5,15 \text {, } \\
30 \text {, and } 45 \text { minutes } \\
\text { contained no penicil- } \\
\text { lin }\end{array}$ \\
\hline $\begin{array}{l}\text { Intra- } \\
\text { venous }\end{array}$ & 10,000 & 5 & 23 & 101 & $\begin{array}{r}5 \\
105\end{array}$ & $\begin{array}{r}5.0 \\
0\end{array}$ & & & & & $\begin{array}{l}\text { Patient } 5 \text { months preg- } \\
\text { nant. Spinal fluid } \\
\text { at } 95 \text { minutes con- } \\
\text { tained no penicillin }\end{array}$ \\
\hline $\begin{array}{l}\text { Intra- } \\
\text { venous }\end{array}$ & 20,000 & 6 & 52 & 153 & $\begin{array}{r}1 \\
10 \\
22 \\
45 \\
125 \\
150 \\
185\end{array}$ & $\begin{array}{c}2.5 \\
0.625 \\
0.156 \\
0.156 \\
0.019 \\
0.007 \\
\text { tr. }\end{array}$ & $\begin{array}{r}60 \\
120 \\
180 \\
240 \\
300 \\
345\end{array}$ & $\begin{array}{l}18.4 \\
14.5 \\
79.6 \\
28.9 \\
25.4 \\
21.6\end{array}$ & \begin{tabular}{|r|}
175.4 \\
129.3 \\
29.1 \\
6.2 \\
1.6 \\
1.9
\end{tabular} & $\begin{array}{l}3,228 \\
5,103 \\
7,424 \\
7,603 \\
7,644 \\
7,688\end{array}$ & Brain tumor \\
\hline $\begin{array}{l}\text { Intra- } \\
\text { venous }\end{array}$ & 20,000 & 7 & 24 & 112 & $\begin{array}{r}5 \\
90 \\
180\end{array}$ & $\begin{array}{c}10.0 \\
0.156 \\
0\end{array}$ & 240 & 124.0 & 160.0 & 19,840 & $\begin{array}{l}\text { Subacute bacterial en- } \\
\text { docarditis }\end{array}$ \\
\hline
\end{tabular}

* All times recorded are from the end of penicillin administration.

t No further urine specimens obtained.

Serum and urine concentrations are recorded only to the time when penicillin was no longer detected. $T r .=$ trace of penicillin. 
TABLE I-Continued

\begin{tabular}{|c|c|c|c|c|c|c|c|c|c|c|c|}
\hline \multirow[b]{2}{*}{ Route } & \multirow[b]{2}{*}{ Dose } & \multicolumn{3}{|c|}{ Subject } & \multicolumn{2}{|c|}{ Serum } & \multicolumn{4}{|c|}{ Urine } & \multirow[b]{2}{*}{$\begin{array}{l}\text { Diagnosis and } \\
\text { remarks }\end{array}$} \\
\hline & & Number & Age & Weight & Time * & Units & Time & Volume & Units & $\begin{array}{l}\text { Units } \\
\text { ex- } \\
\text { creted } \\
\text { (cumu- } \\
\text { lative) }\end{array}$ & \\
\hline $\begin{array}{l}\text { Intra- } \\
\text { venous }\end{array}$ & 20,000 & 8 & $\begin{array}{c}\text { years } \\
48\end{array}$ & $\begin{array}{l}\text { lbs. } \\
133\end{array}$ & $\begin{array}{c}\text { minutes } \\
10 \\
25 \\
50 \\
75 \\
105 \\
150 \\
180\end{array}$ & $\begin{array}{c}\text { per } c c . \\
1.25 \\
0.312 \\
0.078 \\
0.039 \\
0.019 \\
\text { tr. } \\
0\end{array}$ & minutes & $c c$. & per $c c$. & & $\begin{array}{l}\text { Spinal fluid at } 135 \text {, } \\
165 \text {, and } 195 \text { min- } \\
\text { utes contained no } \\
\text { penicillin }\end{array}$ \\
\hline $\begin{array}{l}\text { Intra- } \\
\text { venous }\end{array}$ & 20,000 & 9 & 35 & 124 & $\begin{array}{r}10 \\
20 \\
30 \\
40 \\
50 \\
60 \\
82 \\
100 \\
115 \\
140\end{array}$ & $\begin{array}{c}0.625 \\
0.312 \\
0.156 \\
0.078 \\
0.039 \\
0.039 \\
0.039 \\
0.019 \\
0.007 \\
0\end{array}$ & $\begin{array}{r}15 \\
30 \\
45 \\
60 \\
75 \\
105 \\
120 \\
150 \\
165 \\
210 \\
240 \\
300 \\
360 \\
660\end{array}$ & $\begin{array}{r}4.2 \\
9.4 \\
21.0 \\
55.4 \\
93.0 \\
113.9 \\
34.9 \\
34.8 \\
14.2 \\
40.1 \\
37.2 \\
132.7 \\
96.9 \\
217.4\end{array}$ & $\begin{array}{c}0 \\
125.0 \\
250.0 \\
31.2 \\
2.68 \\
1.56 \\
1.83 \\
3.12 \\
3.13 \\
1.56 \\
0.78 \\
0.20 \\
0.08 \\
0.04\end{array}$ & $\begin{array}{c}0 \\
1,174 \\
6,424 \\
8,152 \\
8,478 \\
8,655 \\
8,734 \\
8,847 \\
8,891 \\
8,954 \\
8,984 \\
9,011 \\
9,019 \\
9,028\end{array}$ & Boeck's sarcoid \\
\hline $\begin{array}{l}\text { Intra- } \\
\text { venous }\end{array}$ & 40,000 & 10 & 33 & 155 & $\begin{array}{r}1 \\
3 \\
9 \\
13 \\
25 \\
42 \\
65 \\
80 \\
95 \\
120 \\
140 \\
180\end{array}$ & $\begin{array}{l}2.5 \\
1.25 \\
0.625 \\
0.312 \\
0.156 \\
0.078 \\
0.078 \\
0.039 \\
0.019 \\
0.007 \\
0.007 \\
0\end{array}$ & $\begin{array}{l}125 \\
225 \\
330 \\
400 \\
480\end{array}$ & $\begin{array}{r}181.0 \\
170.0 \\
36.0 \\
36.0 \\
52.0\end{array}$ & $\begin{array}{l}80.0 \\
1.25 \\
0.312 \\
0.625 \\
0.078\end{array}$ & $\begin{array}{l}14,480 \\
14,692 \\
14,702 \\
14,724 \\
14,728\end{array}$ & Chronic osteomyelitis \\
\hline $\begin{array}{l}\text { Subcuta- } \\
\text { neous }\end{array}$ & 10,000 & 12 & 21 & 163 & $\begin{array}{r}5 \\
20 \\
35 \\
60 \\
85 \\
120 \\
150 \\
190 \\
255 \\
300\end{array}$ & $\begin{array}{c}0 \\
0 \\
0 \\
\text { tr. } \\
\text { tr. } \\
0.007 \\
0.007 \\
0.007 \\
0.007 \\
\text { tr. }\end{array}$ & $\begin{array}{l}120 \\
300 \\
540 \\
660\end{array}$ & $\begin{array}{l}285.0 \\
135.0 \\
205.0 \\
123.0\end{array}$ & $\begin{array}{c}1.25 \\
10.0 \\
1.25 \\
0.078\end{array}$ & $\begin{array}{r}356 \\
1,706 \\
1,962 \\
1,972\end{array}$ & Rheumatic fever \\
\hline $\begin{array}{c}\text { Subcuta- } \\
\text { neous }\end{array}$ & 10,000 & 13 & 30 & 127 & $\begin{array}{r}5 \\
15 \\
40 \\
60 \\
80 \\
100 \\
115 \\
135 \\
155 \\
180 \\
205\end{array}$ & $\begin{array}{c}0 \\
0 \\
0 \\
0 \\
0 \\
0 \\
\text { tr. } \\
\text { tr. } \\
\text { tr. } \\
\text { tr. } \\
0\end{array}$ & $\begin{array}{r}70 \\
250 \\
380 \\
540 \\
720 \\
1,470\end{array}$ & $\begin{array}{r}182.0 \\
70.0 \\
85.0 \\
91.0 \\
112.0 \\
226.0\end{array}$ & $\begin{array}{c}5.0 \\
80.0 \\
20.0 \\
2.5 \\
0.312 \\
0\end{array}$ & $\begin{array}{l}900 \\
6,500 \\
8,200 \\
8,427 \\
8,462 \\
8,462\end{array}$ & Normal subject \\
\hline $\begin{array}{c}\text { Intramus- } \\
\text { cular }\end{array}$ & 10,000 & 1 & 36 & 139 & $\begin{array}{r}5 \\
30 \\
60 \\
90 \\
120 \\
150\end{array}$ & $\begin{array}{l}0.039 \\
0.078 \\
0.078 \\
0.039 \\
0.007 \\
0\end{array}$ & & & & & Chronic osteomyelitis \\
\hline
\end{tabular}


TABLE I-Continued

\begin{tabular}{|c|c|c|c|c|c|c|c|c|c|c|c|}
\hline \multirow[b]{2}{*}{ Route } & \multirow[b]{2}{*}{ Dose } & \multicolumn{3}{|c|}{ Subject } & \multicolumn{2}{|c|}{ Serum } & \multicolumn{4}{|c|}{ Urine } & \multirow[b]{2}{*}{$\begin{array}{l}\text { Diagnosis and } \\
\text { remarks }\end{array}$} \\
\hline & & Number & Age & Weight & Time* & Units & Time & Volume & Units & $\begin{array}{l}\text { Units } \\
\text { ex- } \\
\text { creted } \\
\text { (cumu- } \\
\text { lative) }\end{array}$ & \\
\hline $\begin{array}{l}\text { Intramus- } \\
\text { cular }\end{array}$ & 10,000 & 14 & $\begin{array}{l}\text { years } \\
28\end{array}$ & $\begin{array}{l}\text { lbs. } \\
154\end{array}$ & $\begin{array}{c}\text { minutes } \\
5 \\
12 \\
25 \\
45 \\
70 \\
100 \\
120 \\
135 \\
160 \\
185\end{array}$ & $\begin{array}{c}\text { per } c \text {. } \\
0.078 \\
0.078 \\
0.078 \\
0.078 \\
0.015 \\
\text { tr. } \\
\text { tr. } \\
\text { tr. } \\
\text { tr. } \\
0\end{array}$ & $\begin{array}{l}\text { minutes } \\
100 \\
250 \\
390 \\
1,320\end{array}$ & $\begin{array}{c}c c . \\
415.0 \\
238.0 \\
598.0 \\
380.0\end{array}$ & $\begin{array}{c}\text { per cc. } \\
20.0 \\
5.0 \\
0.156 \\
0\end{array}$ & $\begin{array}{l}8,300 \\
9,490 \\
9,583 \\
9,583\end{array}$ & Rheumatoid arthritis \\
\hline $\begin{array}{l}\text { Intra- } \\
\text { articu- } \\
\text { lar } \\
\text { (knee } \\
\text { joint) }\end{array}$ & 10,000 & 12 & 21 & 163 & $\begin{array}{r}5 \\
15 \\
25 \\
45 \\
55 \\
70 \\
95 \\
130 \\
180 \\
210 \\
240\end{array}$ & $\begin{array}{c}0 \\
0 \\
0.007 \\
0.007 \\
0.007 \\
0.019 \\
0.019 \\
0.019 \\
0.019 \\
0.007 \\
0.007\end{array}$ & $\begin{array}{l}120 \\
240 \\
420 \\
540 \\
780\end{array}$ & $\begin{array}{l}208.0 \\
237.0 \\
190.0 \\
176.0 \\
465.0\end{array}$ & \begin{tabular}{l|}
2.5 \\
5.0 \\
2.5 \\
0.312 \\
0.019
\end{tabular} & $\begin{array}{r}520 \\
1,705 \\
2,108 \\
2,163 \\
2,171\end{array}$ & Rheumatic fever \\
\hline $\begin{array}{l}\text { Intra- } \\
\text { pleural }\end{array}$ & $\begin{array}{c}10,000 \\
.\end{array}$ & 19 & 40 & & $\begin{array}{r}15 \\
30 \\
60 \\
90 \\
120 \\
150 \\
210 \\
255 \\
300 \\
375 \\
435\end{array}$ & $\begin{array}{c}\text { tr. } \\
0.007 \\
\text { tr. } \\
\text { tr. } \\
0.007 \\
0.007 \\
\text { tr. } \\
\text { tr. } \\
0.007 \\
0.007 \\
0.007\end{array}$ & $\begin{array}{r}275 \\
600 \\
1,020\end{array}$ & $\begin{array}{l}525.0 \\
575.0 \\
336.0\end{array}$ & $\begin{array}{l}2.5 \\
0.625 \\
0.625\end{array}$ & $\begin{array}{l}1,312 \\
1,671 \\
1,881\end{array}$ & $\begin{array}{l}\text { Empyema fluid at } 22 \\
\text { hours contained } 0.78 \\
\text { unit per cc. }\end{array}$ \\
\hline $\begin{array}{l}\text { Intra- } \\
\text { pleural }\end{array}$ & 5,000 & 21 & 13 & 78 & $\begin{array}{r}60 \\
120 \\
175 \\
245\end{array}$ & $\begin{array}{l}\mathbf{0} \\
\mathbf{0} \\
\mathbf{0} \\
\mathbf{0}\end{array}$ & $\begin{array}{r}255 \\
420 \\
660 \\
1,500\end{array}$ & $\begin{array}{l}407.0 \\
228.0 \\
131.0 \\
173.0\end{array}$ & \begin{tabular}{l|}
5.0 \\
0.625 \\
0.312 \\
0.039
\end{tabular} & $\begin{array}{l}2,035 \\
2,177 \\
2,217 \\
2,224\end{array}$ & Chronic empyema \\
\hline $\begin{array}{l}\text { Intra- } \\
\text { pleural }\end{array}$ & 30,000 & 20 & 16 & 123 & $\begin{array}{r}30 \\
90 \\
135 \\
225 \\
380 \\
460\end{array}$ & $\begin{array}{c}0 \\
0.007 \\
0.019 \\
0.039 \\
0 \\
0.007\end{array}$ & $\begin{array}{r}120 \\
305 \\
465 \\
705 \\
900 \\
1,140\end{array}$ & $\begin{array}{l}129.0 \\
135.0 \\
126.0 \\
183.0 \\
135.0 \\
175.0\end{array}$ & $\begin{array}{l}1.25 \\
10.0 \\
5.0 \\
5.0 \\
1.25 \\
1.25\end{array}$ & $\begin{array}{l}161 \\
1,511 \\
2,141 \\
3,056 \\
3,225 \\
3,444\end{array}$ & $\begin{array}{l}\text { Injected into empy- } \\
\text { ema cavity. At } 24 \\
\text { hours empyema fluid } \\
\text { contained } 3.12 \text { units } \\
\text { per cc. }\end{array}$ \\
\hline$\underset{\text { tract }}{\text { Sinus }}$ & 12,000 & 22 & 21 & 132 & $\begin{array}{r}45 \\
80 \\
110 \\
150\end{array}$ & $\begin{array}{c}0.039 \\
0.019 \\
\text { tr. } \\
0\end{array}$ & $\begin{array}{r}485 \\
720 \\
1,200\end{array}$ & $\begin{array}{l}346.0 \\
112.0 \\
173.0\end{array}$ & $\begin{array}{l}0.312 \\
0.156 \\
0.039\end{array}$ & $\begin{array}{l}108 \\
125 \\
132\end{array}$ & Chronic osteomyelitis \\
\hline $\begin{array}{l}\text { Intra- } \\
\text { bursal } \\
\text { (supra- } \\
\text { patel- } \\
\text { lar) }\end{array}$ & 10,000 & 14 & 28 & 154 & $\begin{array}{r}15 \\
30 \\
50 \\
70 \\
85 \\
115 \\
145 \\
190 \\
\end{array}$ & $\begin{array}{c}0 \\
0 \\
0 \\
0 \\
0 \\
0.007 \\
\text { tr. } \\
0\end{array}$ & $\begin{array}{l}120 \\
240 \\
300 \\
360 \\
480 \\
810\end{array}$ & $\begin{array}{r}363.0 \\
91.0 \\
54.0 \\
324.0 \\
548.0 \\
325.0\end{array}$ & $\begin{array}{c}5.0 \\
20.0 \\
10.0 \\
0.312 \\
0.156 \\
0.078\end{array}$ & $\begin{array}{l}1,815 \\
3,635 \\
4,175 \\
4,276 \\
4,361 \\
4,386\end{array}$ & Rheumatoid arthritis \\
\hline
\end{tabular}


TABLE I-Continued

\begin{tabular}{|c|c|c|c|c|c|c|c|c|c|c|c|}
\hline \multirow[b]{2}{*}{ Route } & \multirow[b]{2}{*}{ Dose } & \multicolumn{3}{|c|}{ Subject } & \multicolumn{2}{|c|}{ Serum } & \multicolumn{4}{|c|}{ Urine } & \multirow[b]{2}{*}{$\begin{array}{l}\text { Diagnosis and } \\
\text { remarks }\end{array}$} \\
\hline & & Number & Age & Weight & Time* & Units & Time & Volume & Units & $\begin{array}{l}\text { Units } \\
\text { ex- } \\
\text { creted } \\
\text { (cumu- } \\
\text { lative) }\end{array}$ & \\
\hline Oral & 10,000 & 15 & $\begin{array}{c}\text { years } \\
17\end{array}$ & $\begin{array}{l}\text { lbs. } \\
149\end{array}$ & $\begin{array}{c}\text { minutes } \\
10 \\
25 \\
40 \\
60 \\
80 \\
100 \\
130 \\
155 \\
180\end{array}$ & $\begin{array}{c}\text { per cc. } \\
0 \\
\text { tr. } \\
0.007 \\
0.007 \\
0.007 \\
0.007 \\
\text { tr. } \\
\text { tr. } \\
0\end{array}$ & $\begin{array}{c}\text { minutes } \\
90 \\
180 \\
270 \\
420 \\
480\end{array}$ & $\begin{array}{c}c c . \\
163.0 \\
114.0 \\
532.0 \\
582.0 \\
280.0\end{array}$ & $\begin{array}{l}\text { per cc. } \\
2.5 \\
5.0 \\
0.078 \\
0 \\
0\end{array}$ & $\begin{array}{r}407 \\
977 \\
1,018 \\
1,018 \\
1,018\end{array}$ & Idiopathic epilepsy \\
\hline Oral & 20,000 & 16 & 21 & 142 & $\begin{array}{r}5 \\
30 \\
70 \\
95 \\
120 \\
165 \\
210\end{array}$ & $\begin{array}{l}0 \\
0 \\
0 \\
0 \\
0 \\
0 \\
0\end{array}$ & $\begin{array}{r}60 \\
300 \\
600 \\
840\end{array}$ & $\begin{array}{l}300.0 \\
432.0 \\
255.0 \\
163.0\end{array}$ & $\begin{array}{c}0.312 \\
1.25 \\
0.039 \\
0\end{array}$ & $\begin{array}{r}93 \\
633 \\
643 \\
643\end{array}$ & Inguinal hernia \\
\hline Oral & 20,000 & 16 & 21 & 142 & $\begin{array}{r}5 \\
18 \\
30 \\
45 \\
65 \\
85\end{array}$ & $\begin{array}{l}0 \\
0 \\
0.007 \\
0.039 \\
0.007 \\
0\end{array}$ & $\begin{array}{l}165 \\
325 \\
365 \\
560 \\
705\end{array}$ & $\begin{array}{r}200.0 \\
243.0 \\
90.0 \\
134.0 \\
127.0\end{array}$ & $\begin{array}{l}2.5 \\
1.25 \\
2.5 \\
0.078 \\
0\end{array}$ & $\begin{array}{r}500 \\
803 \\
1,028 \\
1,038 \\
1,038\end{array}$ & $\begin{array}{l}\text { Given } 4 \text { grams of so- } \\
\text { dium bicarbonate } 10 \\
\text { minutes before ad- } \\
\text { ministration of peni- } \\
\text { cillin }\end{array}$ \\
\hline $\begin{array}{l}\text { Duo- } \\
\text { denal }\end{array}$ & 10,000 & 17 & 42 & 199 & $\begin{array}{r}5 \\
15 \\
30 \\
45 \\
60 \\
75\end{array}$ & $\begin{array}{l}0.007 \\
0.039 \\
0.019 \\
0.007 \\
\text { tr. } \\
0\end{array}$ & $\begin{array}{l}240 \\
330 \\
465\end{array}$ & $\begin{array}{l}557.0 \\
171.0 \\
330.0\end{array}$ & $\begin{array}{l}2.5 \\
1.25 \\
0.019\end{array}$ & $\begin{array}{l}1,392 \\
1,606 \\
1,612\end{array}$ & Menorrhagia \\
\hline $\begin{array}{l}\text { Duo- } \\
\text { denal }\end{array}$ & 20,000 & .18 & 35 & 128 & $\begin{array}{r}5 \\
15 \\
25 \\
35 \\
45 \\
55 \\
80\end{array}$ & $\begin{array}{c}0 \\
0.039 \\
0.039 \\
0.039 \\
0.019 \\
0.019 \\
\text { tr. }\end{array}$ & $\begin{array}{r}55 \\
215 \\
285 \\
495\end{array}$ & $\begin{array}{r}555.0 \\
156.0 \\
73.0 \\
95.0\end{array}$ & $\begin{array}{l}2.5 \\
2.5 \\
2.5 \\
0.625\end{array}$ & $\begin{array}{l}1,387 \\
1,777 \\
1,958 \\
2,017\end{array}$ & Psychoneurosis \\
\hline Rectal & 10,000 & 1 & 36 & 139 & $\begin{array}{r}10 \\
45 \\
90 \\
120 \\
150 \\
180 \\
225 \\
270\end{array}$ & $\begin{array}{c}0 \\
0.007 \\
0.007 \\
0.007 \\
\text { tr. } \\
\text { tr. } \\
\text { tr. } \\
0\end{array}$ & $\begin{array}{l}120 \\
240 \\
360 \\
480 \\
540\end{array}$ & $\begin{array}{r}111.0 \\
80.0 \\
108.0 \\
80.0 \\
135.0\end{array}$ & $\begin{array}{l}2.5 \\
5.0 \\
2.5 \\
2.5 \\
0.625\end{array}$ & $\begin{array}{r}277 \\
677 \\
947 \\
1,147 \\
1,231\end{array}$ & Chronic osteomyelitis \\
\hline Rectal & 20,000 & 1 & 36 & 139 & $\begin{array}{r}5 \\
20 \\
40 \\
60 \\
80 \\
100 \\
120 \\
150 \\
180 \\
240 \\
270 \\
300 \\
360\end{array}$ & $\begin{array}{c}0 \\
0.007 \\
0.007 \\
\text { tr. } \\
0 \\
0.007 \\
0.007 \\
0.007 \\
0.007 \\
\text { tr. } \\
0.007 \\
\text { tr. } \\
0\end{array}$ & $\begin{array}{r}50 \\
120 \\
240 \\
360 \\
480 \\
600\end{array}$ & $\begin{array}{r}454.0 \\
302.0 \\
88.0 \\
135.0 \\
88.0 \\
94.0\end{array}$ & $\begin{array}{l}0.078 \\
0.625 \\
5.0 \\
1.25 \\
1.25 \\
1.25\end{array}$ & $\begin{array}{r}35 \\
223 \\
663 \\
831 \\
941 \\
1,058\end{array}$ & Chronic osteomyelitis \\
\hline
\end{tabular}




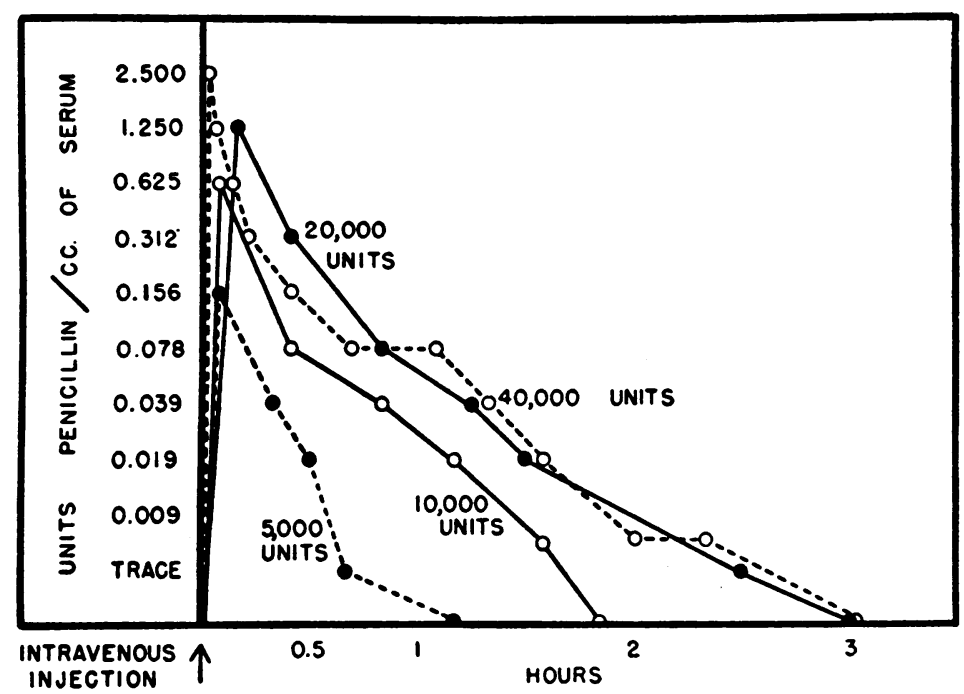

Fig. 2. Serum Concentrations Following Varying Doses of Penicillin

EFFECT OF VARIOUS PARENTERAL ROUTES OF ADMINISTRATION ON SERUM CONCENTRATION AND URINARY EXCRETION

In contrast to the immediate rise and very rapid fall in the level of penicillin in the serum following an intravenous injection, were the concentrations obtained after intramuscular and subcutaneous injections (Figure 3). After an intramuscular injection of 10,000 Florey units, there was a rather rapid rise in the serum concentration, which, however, did not reach as high a level as that obtained after an intravenous dose. The concentration in the serum then tended to remain at the peak height for 30 to 45 minutes and

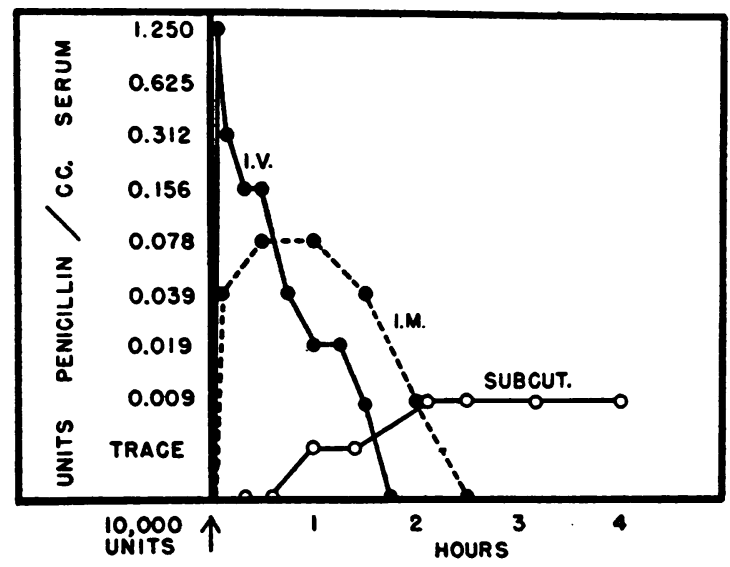

Fig. 3. Concentration of Penicillin in Blood Serum Following Parenteral Injection thereafter decreased gradually. In 2 subjects (Numbers 1 and 14), the last trace was detected at 120 and 160 minutes, respectively. Urinary excretion was not prolonged in Subject 4.

The subcutaneous injection of 10,000 Florey units resulted in a rather prolonged delay in the appearance of penicillin in the blood stream. In 2 subjects (Numbers 12 and 13), it was first detected in the serum at 85 and 115 minutes, respectively. Its concentration in the plasma never reached the high levels obtained by either intravenous or intramuscular injection. Excretion in the urine was definitely delayed.

\section{ABSORPTION AND EXCRETION FROM BODY CAVITIES}

In Subject 12, 10,000 Florey units of penicillin were injected into the right knee joint. Penicillin was first detected in the serum 25 minutes later and reached a maximum height of 0.019 Florey unit per cc. at 70 minutes. The excretion in the urine was similar to that observed after subcutaneous injections in that it was delayed. A total of 21 per cent of the administered dose was found in the urine. At the end of 780 minutes, the knee was again aspirated and the fluid thus obtained was found to contain 0.039 Florey unit per cc.

In another subject (Number 14), 10,000 Florey units were injected into the suprapatellar bursa after it had been aspirated. Penicillin was first detected in the serum at 115 minutes. There was 


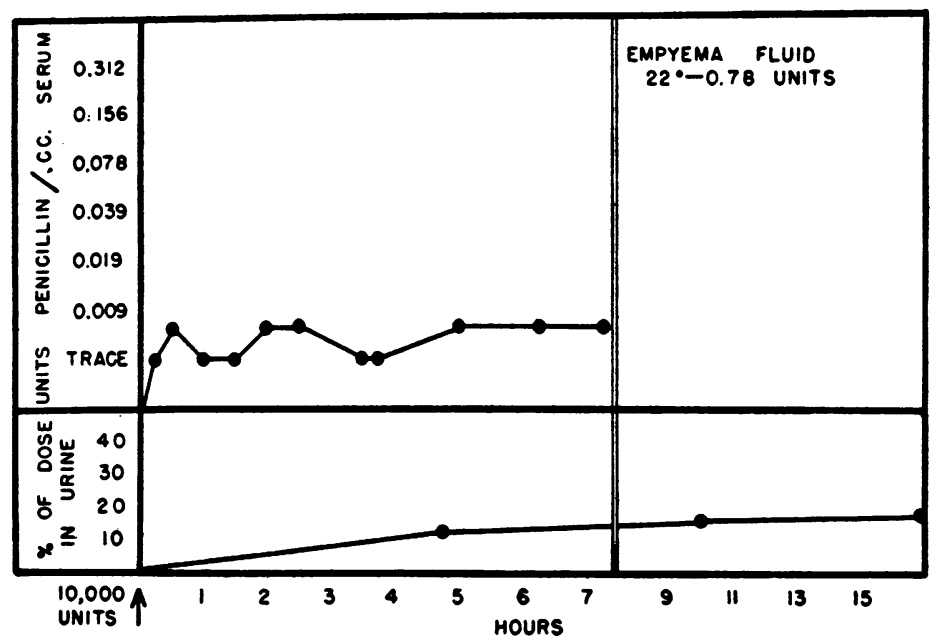

Fig. 4. Absorption and Excretion of Penicillin from Empyema Cavity

a delay in its excretion in the urine, but the total amount excreted was 43 per cent.

Figure 4 shows the results obtained in Subject 19 following the injection of 10,000 Florey units directly into an empyema cavity. The cavity was aspirated and the penicillin then injected through the same needle. Within 15 minutes, a trace of penicillin was present in the serum, and for a total of 420 minutes, all samples of plasma contained a trace to 0.007 Florey unit per cc. The excretion in the urine was somewhat delayed. At the time of surgical drainage, 22 hours after the injection, a sample of the exudate contained 0.78 Florey unit per cc.

Subject 21 received only 5,000 Florey units which were injected through a draining sinus into an old empyema cavity. In this subject, no penicillin was detected in the serum; however, 44 per cent of the dose injected was found to be excreted in the urine. On another occasion, the same subject excreted 42 per cent of 5,000 Florey units which had been injected into the sinus. Following this, the patient received 3 injections of 10,000 Florey units and the subsequent excretion was found to be 33,4 , and 13 per cent, respectively.

One subject (Number 20) who developed an empyema following a lobectomy received 30,000 Florey units in a single dose. There was a rather long delay in the appearance of the substance in the blood stream and likewise a delay in its excretion in the urine. The total excretion amounted to 11 per cent of the administered dose. Aspiration of the cavity 24 hours after injection showed that the exudate still contained 3.12 units per cc.

Similar results were obtained when penicillin was injected into a chronic sinus extending from the left buttock up to the lower pole of the left kidney (Subject 22). Penicillin appeared in the serum in this patient although the urinary excretion amounted to only 1 per cent. This may be partially explained by the fact that after the first 4 hours of this study, the patient moved about in bed, which allowed the penicillin to drain out through the sinus.

\section{ABSORPTION AND EXCRETION AFTER ENTERAL ADMINISTRATION}

The absorption and excretion of penicillin were studied after oral, intraduodenal, and rectal administration of 10,000 to 20,000 Florey units. Figure 5 demonstrates the concentrations obtained in the serum of the 3 subjects (Numbers 17,15 , and 1) following the administration of 10,000 Florey units by the intraduodenal, oral, and rectal routes, respectively. After the intraduodenal administration, there was a greater and more rapid rise in the concentration in the serum than was observed following rectal or oral administration, the curve being similar to that obtained after intravenous or intramuscular injection in that there was a rather abrupt rise, a short duration of the peak concentration, and a rapid 


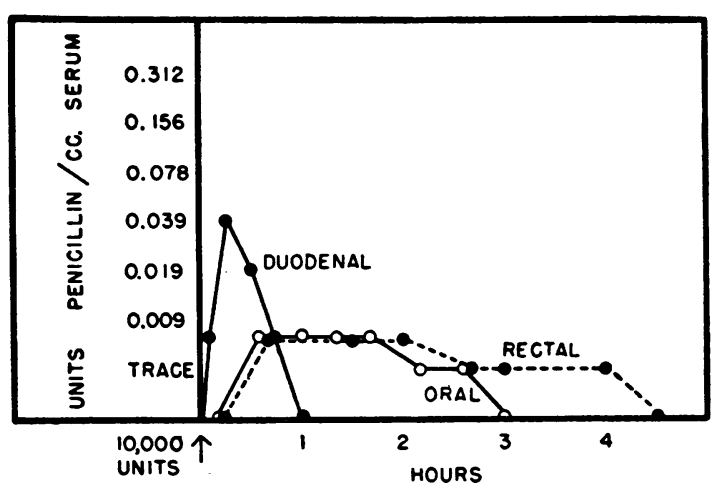

Fig. 5. Concentration of Penicillin in Blood Serum After Enteral Administration

disappearance of penicillin from the blood stream. Although the excretion was apparently more rapid following intraduodenal administration, the total amount found in the urine was of the same order as that found after oral and rectal administration, the amount excreted being 10, 16, and 12 per cent following the oral, intraduodenal, and rectal doses, respectively.

In Subject 16, 20,000 units of penicillin were given by mouth after 12 hours of fasting, and again after a 12-hour fast and the ingestion of 4 grams of sodium bicarbonate, 10 minutes prior to the dose of penicillin. In the first instance, no penicillin appeared in the serum; following sodium bicarbonate, small amounts were detected for a period of 35 minutes. The excretion in the two tests was of the same order, being 3 and 5 per cent of the administered dose.

\section{EFFECT OF RENAL FUNCTION ON EXCRETION}

In this study, 3 patients were given a standard dose of 10,000 units of penicillin intravenously. Brief case histories are presented below.

\section{Subject $A$}

This patient, a female, 34 years old, had been observed over a period of 6 years with chronic progressive renal failure. At the time penicillin was administered, the blood pressure was 180/106. Urine examinations showed a specific gravity of 1.003 to 1.013 , albumin was present in all specimens, and many leukocytes and erythrocytes were seen on microscopic examination. The red cell count was $2,770,000$ and the hemoglobin content was 56 per cent. The non-protein nitrogen was $134 \mathrm{mgm}$. per $100 \mathrm{cc}$., and the urea clearance 10 per cent.

\section{Subject $B$}

This female, 25 years of age, who had entered the hospital because of an attack of acute disseminated lupus erythematosus at the age of 23 , again entered the hospital. The blood pressure was $120 / 78$ and the heart was not enlarged. Anemia was moderate, with $3,810,000$ red cells and a hemoglobin concentration of 70 per cent. The nonprotein nitrogen was $168 \mathrm{mgm}$. per $100 \mathrm{cc}$., and the urea clearance 9 per cent. The specific gravity of the urine ranged from 1.007 to 1.016 and albumin was present in all specimens.

\section{Subject $C$}

A normal subject, 31 years old. (Subject 2, Table I.)

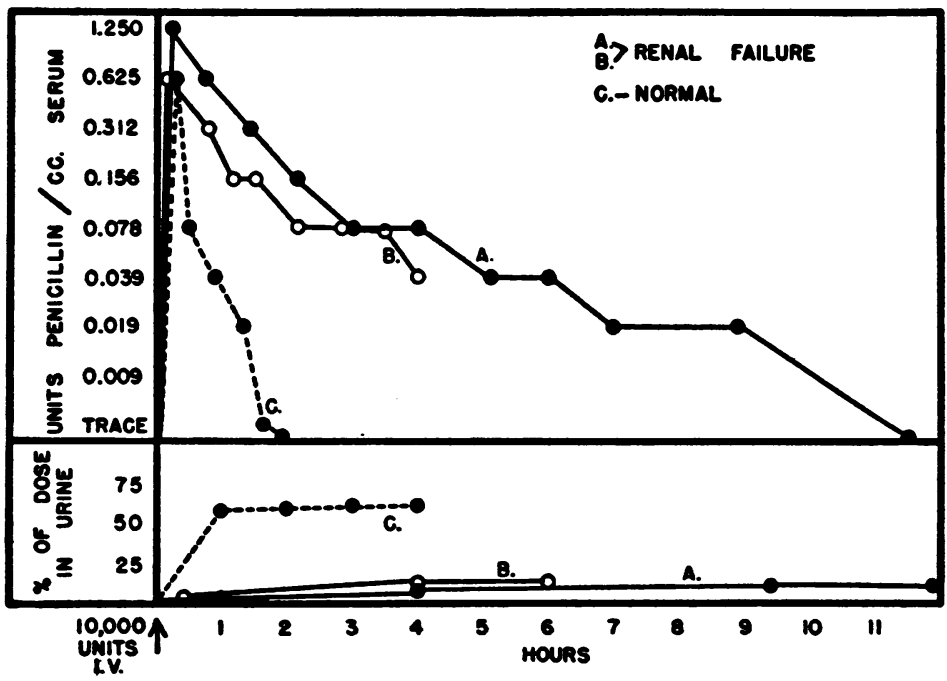

Fig. 6. Effect of Normal and Depressed Renal Function on Excretion of Penicillin 
TABLE II

Distribution of penicillin between blood plasma and red blood cells

\begin{tabular}{|c|c|c|c|c|c|c|c|c|c|c|c|c|c|}
\hline \multirow[b]{2}{*}{ Unknown sample * } & \multirow[b]{2}{*}{ Culture $\dagger$} & \multicolumn{12}{|c|}{ Growth in serial dilutions of unknown samples } \\
\hline & & $\begin{array}{l}\text { Undiluted } \\
\text { sample } \\
0.2 \text { cc. }\end{array}$ & $1: 2$ & 1:4 & $1: 8$ & 1:16 & 1:32 & 1:64 & 1:128 & 1:256 & 1:512 & 1:1024 & 1:2048 \\
\hline Whole blood & $\begin{array}{l}\text { B.B. } \\
\text { B.A.P. }\end{array}$ & $\mathbf{0}$ & 0 & $\mathbf{0}$ & $\mathbf{0}$ & $\mathbf{0}$ & $\mathbf{0}$ & $\begin{array}{l}\mathbf{0} \\
\mathbf{0}\end{array}$ & $\begin{array}{l}\mathbf{0} \\
\mathbf{0}\end{array}$ & $\stackrel{ \pm}{++}$ & + & + & + \\
\hline Serum & $\begin{array}{l}\text { B.B. } \\
\text { B.A.P. }\end{array}$ & $\mathbf{0}$ & 0 & $\mathbf{0}$ & $\mathbf{0}$ & 0 & $\mathbf{0}$ & $\begin{array}{l}\mathbf{0} \\
\mathbf{0}\end{array}$ & $\begin{array}{l}0 \\
0\end{array}$ & $\begin{array}{l}\mathbf{0} \\
\mathbf{0}\end{array}$ & $\stackrel{ \pm}{+t}$ & $\begin{array}{l}++ \\
++\end{array}$ & $+t$ \\
\hline Cells & $\begin{array}{l}\text { B.B. } \\
\text { B.A.P. }\end{array}$ & 0 & $\mathbf{0}$ & $\begin{array}{l}\mathbf{0} \\
0\end{array}$ & $\begin{array}{l}\mathbf{0} \\
\mathbf{0}\end{array}$ & $\stackrel{0}{++}$ & $\begin{array}{l}++ \\
++\end{array}$ & $\begin{array}{l}++ \\
++\end{array}$ & ++ & ++ & ++ & ++ & ++ \\
\hline Hemolyzed cells & $\begin{array}{l}\text { B.B. } \\
\text { B.A.P. }\end{array}$ & & $\mathbf{0}$ & $\begin{array}{l}\mathbf{0} \\
\mathbf{0}\end{array}$ & $\begin{array}{l}\mathbf{0} \\
\mathbf{0}\end{array}$ & $\stackrel{0}{++}$ & ++ & ++ & ++ & ++ & ++ & ++ & ++ \\
\hline
\end{tabular}

* Unknown sample $=$ Defibrinated blood which has been exposed for 19 hours to an unknown amount of penicillin at ice box temperature. Control studies not included in the table have established that 0.0039 Florey unit is the smallest amount of penicillin required to sterilize the culture. The number of units per $0.2 \mathrm{cc}$. of the unknown sample may then be determined by multiplying 0.0039 by the dilution factor. In the whole blood, there are 0.5 Florey unit per 0.2 cc. or 2.5 units per $c c$.

† Culture: B.B. = blood broth. B.A.P. = subculture on blood agar plates

$0=$ no visible growth or hemolysis.

+ and $++=$ degree of growth.

Inoculum $=0.5$ c.c. of blood broth containing 2,700 streptococci.

Figure 6 shows the results obtained in the 2 patients with renal failure, Subjects $A$ and $B$, and also in a normal volunteer, Subject $C$. It is at once apparent that the concentration of penicillin in the serum remained elevated for a prolonged period of time in the 2 subjects with renal failure, whereas all traces of penicillin had disappeared from the blood in the normal subject within 110 minutes.

The prolonged antibacterial effect noted in the serum of the 2 patients is most likely explained by the diminished excretion of penicillin. In the normal subject, 57 per cent of the administered dose was excreted within the first hour, whereas Subject B excreted only 14 per cent in 6 hours, and Subject A, 12 per cent in 13 hours.

\section{DISTRIBUTION OF PENICILLIN BETWEEN BLOOD PLASMA AND RED CELLS}

In order to determine the distribution of penicillin between red cells and blood plasma, whole defibrinated blood containing varying amounts of penicillin was allowed to stand for 2 to 19 hours with occasional agitation. Following this preparation, determinations for penicillin were made on the whole blood, serum, intact cells, and hemolyzed cells.
Table II demonstrates the results obtained in one such experiment. Control observations, using a standard solution of penicillin and made simultaneously with the test, showed that 0.0039 Florey unit was the smallest amount of penicillin which would sterilize the culture. Furthermore, if 0.2 cc. of whole blood, serum, or cells was first added to the standard solution of penicillin and serial dilutions were then made, the end-point was not altered, indicating that these 3 substances do not inhibit the antibacterial action of penicillin.

When the defibrinated whole blood which had been exposed to penicillin for a period of 19 hours was tested, it was found to kill hemolytic streptococci through a dilution of $1: 128$. The serum sterilized the culture through a dilution of $1: 256$, and the cells, both intact and hemolyzed, through a dilution of $1: 8$. Translating this into the number of units per cc., one finds that the whole blood, serum, and cells contained 2.5, 5, and 0.156 Florey units per cc., respectively. The fact that the serum contained twice the concentration of the whole blood sample suggested that the cells contained little or no penicillin.

In Table III, the results of several experiments are recorded; it is apparent that the plasma trapped in the cell mass (5) does account for a significant part of the penicillin detected in the 
TABLE III

Distribution of penicillin between red blood cells and blood plasma

\begin{tabular}{r|r|r|r|r|c|c}
\hline \hline & & \multicolumn{5}{|c}{ Units of penicillin per cc. } \\
\cline { 3 - 7 } $\begin{array}{c}\text { Hours } \\
\text { exposed } \\
\text { to } \\
\text { peni- } \\
\text { cillin }\end{array}$ & $\begin{array}{c}\text { Hem- } \\
\text { ato- } \\
\text { crit }\end{array}$ & \begin{tabular}{c|c|c|c|c} 
Whole \\
blood
\end{tabular} & Plasma & $\begin{array}{c}\text { Cells } \\
\text { (A) }\end{array}$ & $\begin{array}{c}\text { In cell mass } \\
\text { (accounted } \\
\text { for by } \\
\text { trapped } \\
\text { plasma) } \\
\text { (B) }\end{array}$ & $\begin{array}{c}\text { True con- } \\
\text { centration } \\
\text { in cells } \\
\text { (A-B) }\end{array}$ \\
\hline 2 & 45.2 & 2.5 & 5.0 & 0.312 & 0.187 & 0.125 \\
2 & 45.2 & 2.5 & 5.0 & $0.312 *$ & 0.187 & 0.125 \\
19 & 42.0 & 2.5 & 5.0 & 0.156 & 0.174 & -0.018 \\
19 & 42.0 & 10.0 & 10.0 & 0.625 & 0.438 & 0.187 \\
14 & 45.0 & 5.0 & 10.0 & 1.250 & 0.373 & 0.877 \\
14 & 45.0 & 1.25 & 2.5 & 0.312 & 0.093 & 0.219 \\
\hline
\end{tabular}

* Cells hemolyzed before determination of concentration of penicillin.

cells. The true concentration in the cells, as listed in the last column, shows that the amount of penicillin that penetrates the cells is exceedingly small and is usually less than 10 per cent of the plasma concentration.

\section{DISCUSSION}

Few studies on the absorption and excretion of penicillin have been made in man. Florey (3) found that no deleterious effects occurred after the intravenous injection of $200 \mathrm{mgm}$. (about 8,000 Florey units). This was the largest amount administered as a single dose. Following the injection, the initial high value of penicillin in the blood declined to a discernible trace 125 minutes later. Furthermore, the excretion of penicillin in the urine was invariably less than the administered dose. In 2 subjects, 50 and 68 per cent of the antibacterial activity was found in the urine.

In the studies reported here, penicillin was injected intravenously, in doses ranging from 5,000 to 40,000 Florey units. The results agree with the observations of Florey (3) in that penicillin was detected in the peripheral blood and that the active substance was excreted in the urine in amounts less than the dose injected. The concentration reached in the blood plasma was related to the size of the dose injected, the highest concentration being 10 Florey units per cc. of serum. Following the initial rise in the serum concentration, there was a rapid fall. The rapid clearing of the substance from the blood plasma is explained by its excretion in the urine.
Of interest are the results obtained when penicillin was administered by enteral routes. The absorption following oral administration was poor, as demonstrated both by low concentrations obtained in the blood plasma and by the small amounts excreted in the urine. In the 3 tests where penicillin was given orally, the average excretion was 8.6 per cent. The statement (3) that acid destroys penicillin may account for these results; however, in Subject 16, the administration of alkali just prior to ingestion of penicillin did not significantly alter its absorption or excretion. It seems unlikely from these limited observations that oral administration will give adequate concentrations in the blood plasma for the treatment of infections.

Absorption from the intestine was greatest following intraduodenal administration. Here the penicillin appeared in the blood plasma and reached its maximum level within 5 to 15 minutes after the injection. The curve of plasma concentrations was similar to that obtained after intramuscular injection. The excretion in the urine averaged 18 per cent of the administered dose.

Rectal absorption of penicillin was poor, the maximum concentration in the blood plasma being 0.007 Florey unit per cc. following the injection of either 10,000 or 20,000 Florey units. Excretion in the urine was also low, averaging 11 per cent of the injected dose. This decreased excretion may be due entirely to poor absorption; however, Florey (3) observed that feces inactivate penicillin, and more recently it has been demonstrated that extracts of Escherichia coli will inhibit the action of penicillin (6).

The fact that penicillin may be recovered in the urine suggested that the rapid fall in serum concentration following intravenous injection was due primarily to excretion by the normal kidney. This was well demonstrated to be the case in those subjects in whom frequent collections of urine were made. In such subjects, the largest amount of penicillin was excreted during the first hour after the intravenous injection. Indeed, the substance appeared to act as a diuretic in these experiments.

From the study of the 2 subjects with renal failure, further evidence was obtained in support of the view that the chief factor causing the rapid clearing of blood is the excretion in the urine. 
In these patients, a relatively high concentration of penicillin was maintained in the blood plasma for as long as 9 hours after the intravenous injection of 10,000 Florey units.

Little is known concerning the distribution of penicillin in the body fluids. Florey (3) demonstrated in animals that after an intravenous injection, penicillin could be detected in whole blood, bile, and saliva, but not in tears or pancreatic juice. In the present studies, after a single intravenous injection of 10,000 to 20,000 Florey units, no penicillin was found in the spinal fluid. In one subject with osteomyelitis, who was being treated with a constant intravenous drip of 2,500 to 3,000 Florey units per hour, no penicillin was detected in the spinal fluid after 24 hours of such therapy. None was found in the tears or saliva of this patient. However, penicillin injected intrathecally (7) was absorbed and excreted in the urine both in normal subjects and in patients with meningitis. No observations have been made concerning the diffusion of penicillin from the blood stream into the spinal fluid in subjects with meningitis.

The studies on the distribution of penicillin between blood plasma and erythrocytes showed that minimal quantities penetrated the red cells.

The observations made by Florey (3), and confirmed by these studies, that the excretion of penicillin in the urine was always less than the amount administered suggests that it was destroyed or inactivated in the body. In general, after intravenous injection, excretion in the urine accounted for about 60 per cent of the administered dose. When penicillin was administered by routes that result in slow absorption, the percentage recovery in the urine was even lower and, further, in those patients with renal failure, the total excretion was extremely low. These latter two observations support the view that penicillin is inactivated in the body.

The cause of this apparent loss of penicillin is not explained. This study showed that red cells and plasma did not inhibit its action. Further, its incubation with slices of liver, kidney, spleen, brain, muscle, lymph gland, intestine, lung, and bile, caused no destruction of the penicillin (3). Although elevated temperatures will destroy penicillin activity, it is unlikely that body temperatures cause a significant loss of penicillin during the short period it remains in the body.

The maintenance of an adequate concentration of penicillin in the body is necessary if therapy in man is to be successful. Factors of importance in deciding the size of the dose and the route of administration are the type of infecting organism and the site of infection. Micro-organisms vary in their susceptibility to the action of penicillin (3). In general, hemolytic streptococci and pneumococci are extremely sensitive. From in vitro tests, we have found that as little as 0.0039 Florey unit was required to kill from 1,000 to 100,000 hemolytic streptococci, whereas about 0.03 Florey unit was required to sterilize similar numbers of staphylococci (8). Studies on the bactericidal power of whole blood demonstrated that plasma concentrations of 0.03 and 0.3 Florey unit per cc. were required to cause maximal killing against the hemolytic streptococcus and Staphylococcus aureus, respectively (8). Somewhat lower concentrations in the plasma were associated with a definite bacteriostatic effect.

The location of the infection is of utmost importance in determining the route of administration, since it is evident from the studies reported here that penicillin is excreted rapidly and does not diffuse readily. Thus, if a localized infection is being treated by intravenous therapy, the blood supply to the area must be adequate if sterilization is to be effected. It is advisable, therefore, to give penicillin locally rather than intravenously in infections of the pleural and joint cavities. In generalized infections, such as bacteremia, intravenous or intramuscular therapy is indicated.

\section{SUM MARY}

Data are presented concerning the blood concentration and urinary excretion of penicillin after the administration of 5,000 to 40,000 Florey units by several routes.

Intravenous injection of penicillin resulted in high initial concentration in the blood plasma which was followed by an abrupt fall. Traces of penicillin were found in the blood for 30 to 210 minutes after the injection, the length of time depending on the amount administered. The sharp fall noted in the serum concentration immediately after injection was associated with an increased 
excretion in the urine. The average excretion after intravenous injection was 58 per cent of the administered dose.

Penicillin was rapidly absorbed when given intramuscularly and slowly absorbed after subcutaneous injections. Excretion in the urine was rapid following intramuscular injections and delayed after subcutaneous injections.

Absorption from the body cavities was delayed, and this was reflected in the slow excretion of penicillin by the kidneys. The total amount found in the urine was somewhat lower than that obtained following intravenous injection.' Fluid aspirated from the pleural and joint cavities, 22 and 13 hours after the injection, showed appreciable amounts of penicillin remaining.

Administration of penicillin by enteral routes showed that absorption from the duodenum was rapid, whereas oral and rectal doses were poorly absorbed. These findings may be explained by the inactivating effect on penicillin of acid and $E s$ chericia coli. After oral, intraduodenal, and rectal administration, the average amount excreted in the urine was extremely small.

In the presence of renal failure, penicillin was not excreted rapidly, and as a result, high concentrations were maintained in the blood stream after intravenous injections.

Studies on the distribution of penicillin showed that the substance failed to penetrate the red cells in significant amounts. In general, the average concentration found in erythrocytes was less than 10 per cent of the plasma concentration. No penicillin was found in the spinal fluid, saliva, or tears, in subjects receiving it intravenously.

\section{BIBLIOGRAPHY}

1. Fleming, A., On antibacterial action of cultures of penicillin with special reference to their use in isolation of $B$. influenzae. Brit. J. Exper. Path., 1929, $10,226$.

2. Chain, E., Florey, H. W., Gardner, A. D., Jennings, M. A., Orr-Ewing, J., Sanders, A. F., and Heatley, N. G., Penicillin as a chemotherapeutic agent. Lancet, 1940, 2, 226.

3. Abraham, E. P., Florey, H. W., Chain, E., Fletcher, C. M., Gardner, A. D., Heatley, N. G., and Jennings, M. A., Further observations on penicillin. Lancet, 1941, 2, 177.

4. Rammelkamp, C. H., A method for determining the concentration of penicillin in body fluids and exudates. Proc. Soc. Exper. Biol. and Med., 1942, 51, 95.

5. Chapin, M. A., and Ross, J. F., The determination of the true cell volume by dye dilution, by protein dilution, and with radioactive iron. The error of the centrifuge hematocrit. Am. J. Physiol., 1942, 137, 447.

6. Abraham, E. P., and Chain, E., An enzyme from bacteria able to destroy penicillin. Nature, 1940, 146, 837.

7. Rammelkamp, C. H., and Keefer, C. S., Absorption and excretion of penicillin after intrathecal injection. Am. J. M. Sc., 1943, 205, 342.

8. Rammelkamp, C. H., Unpublished observations. 